\title{
Calidad institucional y competitividad En el comercio y producción de países sudamericanos
}

\author{
Darcy Fuenzalida-O'Shee* - Bárbara Valenzuela-Klagges**
}

\begin{abstract}
RESUMEN
El objetivo del estudio es determinar el efecto de la calidad institucional y competitividad global sobre el flujo comercial de los países Sudamericanos (Argentina, Brasil, Chile, Colombia y Perú) con sus socios comerciales para el período 1996-2007 y, en una segunda etapa, cómo estas variables y el comercio aproximado afectan en la producción. Dado que el comercio, la calidad institucional y competitividad global son variables endógenas, se aplicará un modelo gravitacional ampliado en dos etapas y estimado mediante datos de panel estáticos con efectos fijos y aleatorios. La investigación logra detectar que a menor percepción de la corrupción en entidades públicas y aumento de la democracia y competitividad global en el país, mayores serán el comercio bilateral y productividad de los países sudamericanos. Además, el estudio confirma la importancia del acuerdo multilateral APEC (Foro de Cooperación Económica de Asia-Pacifico) en el comercio exterior de los países en estudio.
\end{abstract}

Palabras claves: Comercio; Calidad Institucional; Competitividad; Modelo Gravitacional; APEC; Sudamérica.

\section{Abstract}

The objective of the study is to determine the effect of institutional quality and global competitiveness on the trade flow of the South American countries (Argentina, Brazil, Chile, Colombia and Peru) with their trading partners for the period 1996-2007 and, in a second stage, How these variables and the approximate trade affect in the production. Given that trade, institutional quality and global competitiveness are endogenous variables, a gravitational model will be applied in two stages and estimated using static panel data with fixed and random effects. The research shows that the lower perception of corruption in public entities and increased democracy and global competitiveness in the country, the greater the bilateral trade and productivity of the South American countries. In addition, the study confirms the importance of the multilateral agreement APEC (Asia-Pacific Economic Cooperation Forum) in the foreign trade of the countries under study.

Keywords: Commerce; Institutional Quality; Competitiveness; Gravitational model; APEC; South America.

* darcy.fuenzalida@usm.cl.

** barbara.valenzuela@upla.cl 


\section{INTRODUCCION}

T os estudios de las últimas décadas referidos al comercio especifican que el crecimiento hacia fuera tiene un impacto positivo sobre el crecimiento económico. Así como Nurkse (1964) se refiere al comercio como el "motor del crecimiento" y Lewis (1978) lo identifica como el "lubricante", Bhagwati (1978) y Krueger (1978) asocian el grado de apertura comercial con un desarrollo exportador tangible positivamente correlacionado con la productividad nacional. Estudios más recientes (Rojas et al, 1997; Sachs y Warner, 1997; Hausmann, Hwang \& Rodrik 2006; Herzer \& Nowak-Lehmann, 2006a; Alcalá \& Ciccone (2004); Herzer \& Nowak- Lehmann, 2006b; Redding \& Venables, 2003; Rodríguez \& López, 2010; Álvarez \& García, 2010; Rodríguez \& VenegasMartínez, 2011; Jiménez, 2012) identifican a las exportaciones como determinantes principales del crecimiento económico, siendo fundamentales las exportaciones de productos industriales.

No obstante, la evidencia empírica revela algunos hechos que la literatura teórica del crecimiento económico aún no justifica. Mientras la literatura económica plantea que la renta nacional está en función de sus recursos disponibles (fuerza laboral, capital físico y capital humano) y de la capacidad productiva con la que estos recursos son utilizados para producir bienes y servicios, en numerosos países la evidencia empírica apunta hacia otros factores determinantes del crecimiento, destacándose características estructurales exógenas y endógenas de una economía que, para Rodrik (2003), son posibles de agrupar en tres categorías: comercio, geografía e instituciones.

Según Sachs (2000 y 2003), la geografía influye en el desarrollo económico y social a través de tres canales básicos: productividad de la tierra, condiciones de salud de las personas y frecuencia e intensidad de los desastres naturales. Moncayo (2004) concluye que las condiciones adversas de geografía física y humana pueden afectar negativamente en el crecimiento de las regiones más rezagadas. Por otra parte, Coulibaly y Fontagné (2006) miden el impacto de los obstáculos geográficos en el comercio Sur-Sur y concluyen que el principal factor determinante de este "Missing Trade" es la geografía. 
Igualmente, los estudios de Frankel y Romer (1999), Naude \& Gries (2009) y Rodríguez-Pose, et al. (2013) destacan aspectos geográficos que influyen en el comercio de un país. Sin embargo, al analizar el paradigma de la geografía en varios países, incluido Australia ${ }^{1}$, Rodrik (2003) concluye que los problemas geográficos pueden solucionarse con buenas instituciones.

Fajnzylber (1988) plantea que en el mercado internacional no solo compiten empresas, también se confrontan sistemas productivos, esquemas institucionales y organismos sociales que integrados conforman una red de vinculaciones con el sistema educativo, tecnológico, laboral, institucional público y privado, entre otros. En su estudio señala que "En las naciones industrializadas el debate en torno al mejoramiento de la competitividad se da en el marco de instituciones cuya legitimidad nadie la cuestiona" (pp.23). En concordancia, Novella (1995) indica que "Los escritos de Posner, Vernon, Hirsch, Krugman y Cohen observan cómo las ventajas no son comparativas (dadas históricamente) sino que pueden ser competitivas (susceptibles de ser creadas)" (pp. 90), siendo la tecnología, la formación de trabajadores y empresarios y el marketing internacional variables decisivas en el comercio internacional. Algunas investigaciones han validado el efecto de la competitividad global sobre las exportaciones (Contreras-Castillo, 1999; Pérez, 2007; García, Gómez y Martínez, 2010 y Gómez, 2013). Por ejemplo, el estudio de Pérez (2007) analiza el desempeño de las empresas exportadoras cubanas y el estudio de García, Gómez y Martínez (2010) considera el beneficio de una estrategia basada en el "made in" para el caso de Galicia, núcleo fundamental de la industria de granito en España. Otras investigaciones han validado el efecto de la competitividad sobre la productividad en países Sudamericanos (Kogan, 2012 y Fonseca et al. 2015, entre otros). Diversos estudios (Borner, Brunetti \& Weder 1992; Mauro, 1995; Sachs \& Warner, 1995; Rodrik, 2003; Edison, 2003; Rodrik, 2005; De Gregorio, 2005; Rodrik, 2008; Alonso, 2012; Rodrik, 2014) han confirmado la influencia de la calidad de las instituciones en el crecimiento económico. 
Dado lo planteado, el objetivo de este estudio es determinar el efecto de la calidad institucional y competitividad global sobre el flujo comercial de los países Sudamericanos (Argentina, Brasil, Chile, Colombia y Perú) con sus socios comerciales para el período 1996-2007 y, en una segunda etapa, cómo estas variables y el comercio aproximado afectan en la producción. El motivo principal para la elección de este período es que se formaliza un proceso integracionista latinoamericano y se incrementa el comercio intraregional, posiblemente provocado por la liberalización del comercio en esta región. A mediados de la década de los noventa, se configuran procesos de globalización y reformas en que se destacan los bloques comerciales MERCOSUR (Mercado Común del Sur) y CAN (Comunidad Andina, incluido Perú, desde 1998); NAFTA (Tratado de Libre Comercio de América del Norte) con la incorporación de México en 1994; APEC con el ingreso de Chile y México y; acuerdos bilaterales de libre comercio, principalmente incentivados por Chile, tales como, Chile - Venezuela (1996), Chile - Colombia (1996), Chile - Canadá (1996) y Chile - México (1998), entre otros.

Como el comercio, la calidad institucional y la competitividad global son variables endógenas, se aplicará un modelo gravitacional ampliado en dos etapas y estimado mediante datos de panel estáticos con efectos fijos y aleatorios, sustentándose en 12.480 observaciones.

El estudio se divide en tres secciones. En la primera sección, se revisarán los estudios y evidencias de los últimos años referidos a calidad institucional, competitividad y producción. En la segunda, se especificará el modelo gravitacional ampliado y adaptado a la realidad sudamericana y su metodología de estimación. Finalmente, se expondrán los resultados, conclusiones y recomendaciones.

La investigación logra detectar que a menor percepción de la corrupción en entidades públicas y aumentos de la democracia y competitividad global de un país, mayores serán el comercio bilateral y producción de los países sudamericanos, permitiendo sugerir medidas comerciales, productivas y públicas. Además, el estudio confirma la importancia del acuerdo multilateral APEC (Foro de 
Cooperación Económica de Asia-Pacifico) en el comercio exterior de los países en estudio.

\section{Calidad institucional, competitividad y PRODUCTIVIDAD}

Los autores que patrocinan la calidad institucional, como factor principal del desarrollo económico, sugieren que la productividad de la tierra y las condiciones de salud pueden mejorarse con desarrollo tecnológico e infraestructura orientados a las necesidades de cada país o región. $\mathrm{Al}$ respecto, la temática sobre calidad institucional abarca varios aspectos, entre ellos, el desarrollo urbano con infraestructura de servicios, incentivos e instituciones públicas adecuadas y el potencial destructivo de los desastres naturales enfrentado con estándares de construcción y prevención. Para Krause (2007), un país que se niega a la competencia institucional pierde los beneficios del progreso y condena a su población a una peor calidad de vida. Por el contrario, los países que asumen su papel en el contexto institucional se ven inducidos por la competencia a modernizar sus instituciones, se benefician de las bondades del comercio, reciben recursos, tecnología e inversiones, promueven una mejor calidad de vida y presentan mayor respeto a los derechos individuales. En relación a América Latina, este autor expone que Haití nunca ha logrado consolidar un diseño mínimo de control institucional; Venezuela ha deteriorado la calidad de su democracia y Argentina, que fuera la gran promesa de la región a comienzos del siglo XX, paso a sumirse en un espiral decreciente de golpes militares y crisis económicas.

Diversos estudios (Borner et al., 1992; Mauro, 1995; Sachs y Warner, 1995; Edison, 2003; Rodrik, 2000; Rodrik, 2003; Rodrik, 2005; De Gregorio, 2005; Rodrik, 2008; De Gregorio, 2008; Carballo, 2010; Alonso y Garcimantin, 2011; Valenzuela-Klagges, 2011; McMillan-Rodrik, 2011; Price 2011, Alonso 2012; Rodrik y Subramanian, 2012; León et al. 2014; Rodrik, 2014) apuntan hacia la influencia de la calidad de las instituciones en el crecimiento económico, ya sea medida como ausencia de corrupción, gestión 
gubernamental eficiente, democracia, regulaciones jurídicas o calidad de la burocracia. Cabe señalar los estudios de Rodrik (2000) y Rodrik y Subramanian (2012) que identifican los sistemas políticos participativos como los más eficaces para el procesamiento y agregación de conocimiento local, constituyéndose la democracia en una "meta-institución" para la construcción de buenas instituciones; León et al. (2014), al demostrar que la corrupción afecta la satisfacción de las personas y; el estudio de Alonso y Garcimantin (2011) que identifica los principales determinantes de la calidad institucional: nivel de desarrollo, equidad en la distribución de la renta, existencia de un sistema impositivo eficaz y nivel educativo, observándose que algunas de las variables tradicionalmente planteadas en la literatura (localización geográfica, fragmentación étnica, tradición jurídica u origen colonial) no tendrían incidencia en la calidad institucional. En cuanto a los estudios enfocados en Latinoamérica, Gregorio (2008) reconoce que las instituciones desempeńan un papel decisivo en el comercio exterior y señala que en América Latina las instituciones son débiles y están sujetas a crisis macroeconómicas recurrentes, aspectos que impiden el crecimiento de los vínculos comerciales; Carballo (2010) identifica la coexistencia de altos niveles de pobreza y altas tasas de crecimiento económico en América Latina, situación desconcertante explicada en parte por la forma de medir la corrupción y su relación con la calidad institucional; Valenzuela-Klagges (2011) observa que la apertura comercial y la calidad institucional medida mediante el índice de Percepción de la Corrupción y el índice de Desarrollo Democrático, tienen un efecto positivo en el comercio bilateral sudamericano y en la producción de la región y; Price (2011) destaca la inseguridad pública como una limitante que afecta a la capacidad de la región para atraer turismo e inversión y, como efecto posterior, la pérdida del capital humano educado y talentoso que emigra de la región.

Dadas las evidencias, mejorar la calidad institucional pública provocaría efectos positivos en una economía. Sin embargo, hay investigaciones que contradicen este planteamiento. Utilizando datos regionales de México para el periodo 2001-2010, Ramírez y 
Sánchez (2013) analizan la relación entre corrupción y crecimiento económico, evidenciando que los resultados de las estimaciones con mínimos cuadrados ordinarios y datos de panel no permiten validar el modelo teórico esperado e incluso sugieren que en presencia de elevada calidad institucional el efecto de la corrupción suele ser positivo sobre el crecimiento. El estudio de Gantman y Dabós (2013), que abarca 121 países y nueve períodos quinquenales (1961-1965 a 2001-2005), concluye que la calidad institucional en su dimensión política no tiene efecto positivo y estadísticamente significativo sobre el crecimiento económico.

En cuanto a la competitividad global y su importancia en el comercio y crecimiento económico, Contreras-Castillo (1999), Yevenes \& Andalaft (2006), Pérez (2007), Olivares (2009), Mayorga \& Martínez (2010), García, Gómez \& Martínez (2010), Overbaugh (2013), Buendía (2013), Gómez (2013) y Yevenes \& Andalaft (2006) coinciden en afirmar que el requisito prioritario para que los países, regiones y empresas se inserten con éxito en los flujos de comercio internacional es la capacidad de acrecentar su competitividad de una manera duradera y sostenible. Precisamente, para Mayorga \& Martínez (2010), los mercados internacionales son competitivos no solo por la disponibilidad de los factores de producción básicos, sino también, por la adaptación y asimilación de nuevos elementos constitutivos de los factores como son la formación, preparación, calificación y especialización de la mano de obra y la asimilación de tecnologías pertinentes al manejo de los fenómenos macroeconómicos que interfieren en la valoración de los capitales financieros. Buendía (2013) concluye que la prosperidad nacional está determinada en última instancia por la competitividad que representa oportunidades de crecimiento nuevas, sobre todo en países cuyas empresas pueden competir en las grandes ligas internacionales. El estudio sugiere que para desarrollar ventajas competitivas, se requiere de la implementación de una agenda país que permita determinar las áreas y prioridades en el mediano y largo plazo unida a la acción conjunta del Estado y del sector empresarial. Por otra parte, al estudiar los efectos de la cultura nacional y el índice 
de competencia global (Sala-i-Martin et al., 2011) en una muestra de países post comunistas, Overbaugh (2013) explica el crecimiento económico irregular entre estos países debido a los distintos niveles de competitividad, observando que la competitividad de un país se ve afectada por la integración global y transferencia de tecnología entre países y la consolidación de empresas en organizaciones transnacionales. Por consiguiente, en este estudio se concluye la estrecha relación existente entre crecimiento económico y competitividad. El alto nivel de desarrollo y competitividad también indica un aumento en el volumen de transacción económica y mayores ganancias potenciales para firmas internacionales.

Para Rosales (2009), lograr la competitividad en sectores cuyo alcance supere el de un recurso natural de bajo grado de elaboración, exige la formación de una masa crítica de recursos humanos calificados que, unida a las ventajas comparativas naturales y a ciertas facilidades mínimas en materia de infraestructura y conectividad, permita atraer talentos nacionales y extranjeros. Varias investigaciones y evidencias son congruente a este análisis (Pérez, 2007; Hinojosa, 2007; Rodríguez et al., 2010; Park y Jang, 2014; entre otros). Hinojosa (2007), por ejemplo, centra su estudio en identificar los problemas fundamentales que confrontan la economía y la sociedad peruana y sus barreras institucionales, sugiriendo un proceso económico e institucional y un plan de medidas para mejorar sustancialmente los niveles de competitividad internacional, con el objetivo de incrementar el bienestar económico y social del país. El estudio de Park y Jang (2014) utiliza un modelo gravitacional, mediante un sistema de datos de panel, determinando un efecto positivo de la competitividad en el flujo turístico.

\section{EsPeCIFICACIÓN DEL MODELO}

En esta investigación se utilizará un modelo gravitacional ampliado y adaptado a la realidad del Cono Sur Americano para el período 1996 - 2007, con un método en dos etapas similar al formulado por Martínez-Zarzoso y Doyle (2007), con el propósito de examinar la relación entre productividad y comercio y determinar los efectos de 
la calidad institucional y competitividad global en dicha relación.

Los primeros autores que utilizaron el modelo gravitacional para analizar el comercio internacional fueron Tinbergen (1962), Pöyhönen (1963) y Linneman (1966). Tinbergen (1962) propuso una ecuación de gravedad para explicar los niveles de comercio de un país en términos del tamaño de su economía y la de sus socios comerciales (medidos por PNB o PIB) y distancia entre ellos. Linnemann (1966) agregó las variables poblacionales del país exportador e importador para reflejar el desempeño de las economías de escala. Los estudios de Anderson (1979), Bergstrand (1985) y Helpman \& Krugman (1985) derivaron ecuaciones gravitacionales de modelos de comercio internacional basados en la diferenciación del producto y en el rendimiento a escala creciente, dando sustento teórico riguroso. Mátyás (1997); Chen \& Wall (1999); Bayoumi y Eichengreen (1997); Breuss y Egger (1999), Egger (2000), Marto (2007), entre otros, suministran especificaciones econométricas de la ecuación gravitacional.

En la primera etapa, la ecuación especificada (Ecuación 1) tiene como variable dependiente el comercio bilateral total de un país exportador con sus principales socios comerciales, en términos relativos a la paridad de poder adquisitivo del Producto Interno Bruto real ${ }^{2}$ del exportador (Trade/PPPGDP) en logaritmos naturales, siendo determinada como:

$$
\begin{aligned}
\ln \left(\frac{T R A D E}{P P P G D P}\right) & \\
& =\gamma_{i}+x_{j}+\theta_{t}+\beta_{1} \ln \left(P O P_{i t}\right) \\
& +\beta_{2} \ln \left(P_{P O P_{j t}}\right)+\beta_{3} \ln \left(A_{i} A_{j}\right)+\beta_{4} \ln \left(\text { Dist }_{i j}\right)+\beta_{5} I d_{i j}+\beta_{6} F_{i j}+\beta_{7} I P C_{i j}+\beta_{8} I D D_{i j} \\
& +\beta_{9} C G_{i j}+\beta_{10} M E R C O S U R_{i j}+\beta_{11} A P E C_{i j}+U_{i j} \\
& \text { (Ecuación l) }
\end{aligned}
$$

\section{Donde:}

i denota al exportador, $j$ al importador y t el año,

POP es población; A es área; Dist es la distancia (Km.) entre las capitales $^{3}$ y las variables ficticias que toman valor uno cuando el exportador (i) e importador (j):

Id, comparten el mismo idioma; 
F, comparten frontera en común;

IPC, tienen un Índice de Percepción de la Corrupción mayor que 5; IDD, tienen un Índice de Desarrollo Democrático mayor que 5; CG, tienen un Índice de Competitividad Global mayor a 4,5; MERCOSUR participan en el MERCOSUR (incluyendo Chile); APEC participan en el Foro de Cooperación Económica de Asia Pacífico.

Para medir los efectos de la calidad institucional, se incluyen en el modelo los índices Percepción de la Corrupción y Desarrollo Democrático El Índice de Percepción de la Corrupción es desarrollado por la Fundación Konrad Adenauer y Polilat (www.idd-lat.org) y mide, en una escala de cero (percepción de muy corrupto) a diez (percepción de ausencia de corrupción), los niveles de percepción de corrupción en el sector público en un país determinado, sustentado en diversas encuestas a expertos y empresas. En el 2014, por ejemplo, Dinamarca toma valor 9,2, alcanzando el primer lugar de ausencia de corrupción, mientras que Corea del Norte toma el valor 0,8 , reflejando alta corrupción. Por lo que, socios comerciales con menor percepción de la corrupción, es decir, mayor a 5 en el Índice de Percepción de la Corrupción toman valor uno en el modelo.

El Índice de Desarrollo Democrático (IDD-Lat) elaborado por la Fundación Adenauer (www.transparency.org) considera 31 indicadores agrupados en 4 dimensiones, midiendo las siguientes variables: legalidad del régimen democrático; respeto de derechos políticos y libertades civiles; calidad institucional y grado de eficiencia política y ejercicio del poder efectivo para gobernar. Cada dimensión y cada uno de sus componentes son valuados de 0 a 10. El máximo puntaje corresponde al país con mejor desarrollo democrático. En el 2014, Chile presenta un puntaje de 9,7, alcanzando el primer lugar en Latinoamérica, mientras que Venezuela 2,6, siendo el peor evaluado en la zona ${ }^{4}$.

La competitividad de un país se representa en el modelo con el Índice de Competitividad Global publicado por el Foro Económico Mundial, el cual mide cómo utiliza un país los recursos de que dispone y la capacidad que tiene para proveer a sus habitantes 
un alto nivel de prosperidad. Este índice analiza 12 variables: instituciones; infraestructuras; entorno macroeconómico; salud y educación primaria; educación superior y formación; eficiencia del mercado de bienes; eficiencia del mercado laboral; desarrollo del mercado financiero; preparación tecnológica; tamaño del mercado; sofisticación en materia de negocios e innovación. En el 2014, por ejemplo, Suiza alcanza el primer lugar de competitividad global con un puntaje de 5,67, mientras que Chad alcanza el lugar 148, con un puntaje de 2,85, reflejando baja competitividad.

En la segunda etapa, la ecuación planteada (Ecuación 2) relaciona productividad y comercio, utilizando como proxy el comercio estimado en la ecuación anterior:

$$
\ln \left(\frac{P P P G D P_{c}}{L_{c}}\right)=\alpha_{0}+\alpha_{1} \ln \left(T_{R A D E_{c}}\right)+\alpha_{2} \ln \left(P O P_{c}\right)+\alpha_{3}\left(I P C_{c}\right)+\alpha_{4}\left(C G_{c}\right)+\mu_{c}
$$

\section{(Ecuación 2)}

Siendo:

PPPGDPc la productividad por trabajador y Lc fuerza productiva en el país c.

TRADE representa la medida de apertura comercial que viene dada por la predicción obtenida a partir de la ecuación anterior (Ecuación $1)$.

POP es población y las variables ficticias: IPC que denota la calidad institucional, especificado en esta segunda ecuación por el Índice de Percepción de la Corrupción, el cual toman valor uno cuando el exportador (i) e importador (j) tienen un Índice de Percepción de la Corrupción mayor que 5 y CG que denota el Índice de Competitividad Global, el cual toman valor uno cuando el exportador (i) e importador (j) tienen un índice de Competencia Global mayor a 4,5.

Se estimarán las ecuaciones ( 1 y 2 ) mediante datos de panel estáticos con efectos fijos y aleatorios y, como parámetro comparativo, regresión agrupada pool (OLS) con mínimos cuadrados ordinarios en Ecuación 1 para los países Sudamericanos Argentina, Brasil, Chile, Colombia y Perú con sus principales socios comerciales 
durante período 1996-2007. El total de observaciones por año son 1140 , constituyéndose un total de 12480 observaciones.

\section{Resultados, Conclusiones y Recomendaciones.}

En la tabla 1 se muestran los resultados de la Ecuación 1 mediante regresión agrupada pool (OLS) con mínimos cuadrados ordinarios y datos de panel efectos fijos y aleatorios, siendo posible observar que los coeficientes de las variables presentan signos esperados y la mayoría son estadísticamente significativos. La población del exportador, áreas y la distancia geográfica entre socios comerciales tienen un efecto negativo en el comercio. La población del importador; idioma y frontera en común; miembros del APEC; democracia, medido por el Índice de Desarrollo Democrático; la transparencia Institucional entre socios comerciales, medido por el Índice de Percepción de la Corrupción (IPC), y la competitividad global del país, medido por el Índice de Competitividad Global afectan positiva y directamente en el comercio del país. Cabe señalar que el coeficiente de la variable APEC es positivo y significativo, en contraste con la falta de significatividad del coeficiente de la variable MERCOSUR.

El coeficiente de determinación en la estimación de la Ecuación 1 alcanza el 0,39 mediante pool con MCO; 0,69 en datos de panel con efectos fijos y 0,68 en datos de panel con efectos aleatorios (Tabla 1).

Los resultados mediante efectos aleatorios y expuestos en la Tabla 1, muestran coeficientes significativos semejantes a los observados en regresión agrupada pool (ecuación 1), pero con diferencias en sus valores. Al aplicar la prueba de Breusch y Pagan el p-value (Prob $>$ chi2 $=0.000$ ) nos indica que podemos rechazar la hipótesis nula, por lo tanto, los efectos aleatorios son relevantes y es preferible usar la estimación de efectos aleatorios en vez de la agrupada pool. Por otra parte, la prueba $\mathrm{F}$ de significatividad de los efectos fijos se aplica reportando $\mathrm{p}$-value (Prob $>\mathrm{F}=0.000$ ) y rechazando la hipótesis nula, por lo que es preferible usar el método de efectos fijos en vez del modelo agrupado pool. 
Los resultados obtenidos al aplicar la prueba de Breusch y Pagan para efectos aleatorios y la prueba $\mathrm{F}$ de significatividad para efectos fijos, confirman que tanto efectos aleatorios como efectos fijos son mejores que el modelo agrupado pool. Con el propósito de comparar las diferencias entre el coeficiente de efectos fijos y aleatorio, se aplica la prueba de Hausman, obteniendo un Prob $>$ Chi2 $=0.0031$. Por Consiguiente, se ratifica que el método de efectos fijos es más conveniente que efectos aleatorios.

Tabla No 1. Resultados Estimación Ecuación 1 (Pool MCO y Datos de Panel con Efectos Fijos y Aleatorios)

\begin{tabular}{lcll}
\hline \multicolumn{1}{c}{ Variables } & POOL MCO & $\begin{array}{l}\text { EFECTOS } \\
\text { FIJOS }\end{array}$ & $\begin{array}{l}\text { EFECTO } \\
\text { ALEATORIO }\end{array}$ \\
\hline $\mathrm{C}$ & $-8.2^{* * *}(14.8)$ & & $-8.0^{* * *}(14.6)$ \\
\hline Población i & $-0.2^{* * *}(10.2)$ & $-0.2^{* * *}(9.6)$ & $-0.2^{* *}(9.4)$ \\
\hline Población j & $0.4^{* * *}(24.2)$ & $0.5^{* * *}(5.2)$ & $0.38^{* * *}(22.2)$ \\
\hline Área (i)*Área(j) & $-0.03^{* * *}(2.1)$ & & $-0.03^{* * *}(2.1)$ \\
\hline Distancia Geográfica & $-0.4^{* * *}(9.0)$ & & $-0.4^{* * *}(8.8)$ \\
\hline Idioma en común & $0.2^{* * *}(2.8)$ & & $0.2^{* * *}(2.8)$ \\
\hline Frontera en común & $0.93^{* * *}(9.6)$ & & $0.94^{* * *}(9.8)$ \\
\hline MERCOSUR & $-0.06(0.4)$ & $-0.06(0.4)$ & -0.06 \\
\hline APEC & $0.28^{* * *}(2.6)$ & $0.3^{* * *}(3.2)$ & $0.28^{* * *}(2.6)$ \\
\hline IDD & $0.11^{* *}(2.1)$ & $0.9^{* *}(1.9)$ & $0.11^{* *}(2.2)$ \\
\hline IPC & $0.28^{* * *}(3.4)$ & $0.28^{* * *}(2.8)$ & $0.28^{* * *}(2.8)$ \\
\hline CG & $0.09^{* *}(1.9)$ & $0.08^{* *}(1.8)$ & $0.08^{* *}(1.8)$ \\
\hline R2 & $\mathbf{0 . 3 9}$ & $\mathbf{0 . 6 9}$ & $\mathbf{0 . 6 8}$ \\
\hline
\end{tabular}

Nota: Se expresan en la tabla el valor del coeficiente y estándar de error entre paréntesis, respectivamente. La alta significatividad del coeficiente se expresa con *** (probabilidad de error 0\%); ** (probabilidad de error entre 1 y $2,5 \%$ ) y * (probabilidad 2,5\%a 5\%).

Dado que el método de efectos fijos es más conveniente que efectos aleatorios y agrupado pool para la ecuación 1, se continuará utilizando este método para la Ecuación 2. 
En la tabla 2 se exponen los resultados de la Ecuación 2, observándose que los coeficientes de las variables apertura comercial (medido por la variable TRADE), población, calidad institucional y competitividad presentan efectos positivos y significativo. Al igual que lo planteado por los autores Barro (1999) y Valenzuela-Klagges (2011), los resultados indican que la calidad institucional tiene un efecto beneficioso sobre el crecimiento económico sudamericano y que a mayores niveles de apertura comercial mayor es el incremento en la productividad en los países de Latinoamericana.

El coeficiente de determinación en la estimación de la Ecuación 2 mediante datos de panel con efectos fijos es 0,72 .

Este estudio permite concluir que el desarrollo democrático tiene efectos positivos en la apertura comercial sudamericana, por lo que se recomienda reforzar el respeto al derecho público y libertades civiles, tales como, libertad de prensa, derecho de los ciudadanos a mantenerse informados y a crear sindicatos. Se confirma lo planteado por Rodrik (2000) y Rodrik y Subramanian (2012) al identificarse los sistemas participativos como los más eficaces, sugiriendo posicionar a la democracia como una "meta institucional" que permita construir buenas instituciones. En procesos de crisis o desaceleración económica, un país necesita instituciones fuertes y la democracia puede ayudar a reforzarlas, como también, a fomentar el comercio y, por ende, la producción nacional. 
Tabla No 2. Resultado Estimación Ecuación 2, Datos de Panel Efectos Fijos

\begin{tabular}{ll}
\hline Variables & EFECTOS FIJOS \\
\hline TRADE & $0.32^{* * *}(3,6)$ \\
\hline POP & $0.04^{*} \quad(2.2)$ \\
\hline IPC & $0.08^{* * *}(2,4)$ \\
\hline CG & $0.02^{* *}(1.8)$ \\
\hline R2 & $\mathbf{0 . 7 2}$ \\
\hline
\end{tabular}

Nota: Se expresan en la tabla el valor del coeficiente y estándar de error entre paréntesis, respectivamente. La alta significatividad del coeficiente se expresa con ${ }^{* * *}$ (probabilidad de error $0 \%$ ); ** (probabilidad de error entre 1 y 2,5\%) y* (probabilidad 2,5\%a 5\%).

Además, los resultados permiten concluir que la apertura comercial, calidad institucional y competitividad global tienen un efecto positivo en la productividad de la región. A mayor percepción en el sector público, mayor es el impacto negativo en la apertura comercial y en la producción nacional, siendo fundamental aplicar medidas, procedimientos y leyes que garanticen la transparencia y probidad en los procesos de gestión pública. Esta evidencia es congruente con lo planteado por Alonso (2012) para el caso espańol, al concluir que el marco institucional, es decir, el “... conjunto de instituciones que rigen la vida colectiva estableciendo reglas, normas, rutinas y valores, condicionan la respuesta de los agentes" (pp.77) debe mejorar para que esté en condiciones de crecer y competir con los países miembros del OCDE y Unión Europea.

La competitividad global de un país sudamericano influye positivamente en su apertura comercial y producción. Esta conclusión sugiere que los países sudamericanos que se propongan aumentar sus exportaciones y, por ende, su productividad deberán perfeccionar o renovar la infraestructura portuaria, vial y férrea; establecer un entorno macroeconómico estable; garantizar calidad y total cobertura en salud y educación primaria; optimizar el mercado de bienes y financiero; reformar el mercado laboral con condiciones 
flexibles, adaptativas a la realidad y que potencien las habilidades de cada ciudadano y promover la innovación en diferentes sectores productivos.

Además, el estudio confirma la importancia del acuerdo multilateral APEC en la apertura comercial de los países en estudio y la falta de liderazgo del MERCOSUR en la apertura comercial e integración sudamericana.

\section{Notas de Página}

${ }^{1}$ Australia es uno de los pocos países ricos a pesar de sus grandes áreas tropicales, desiertos y suelos áridos, y con un clima extremadamente variable.

${ }^{2}$ Fuente: WDI Banco Mundial (CD), Base de Dato 2012 (PPP GDP; Población, Área y fuerza productiva) y ALADI, indicadores estadísticos (Comercio bilateral, TRADE).

${ }^{3}$ Fuente: www.wcrl.ars,gov/cec/java/lat-long.htm

${ }^{4} \mathrm{http}: / /$ www.idd-lat.org/2014/downloads/idd-lat-2014.pdf.

BiBLIOGRAFÍA

ALCALÁ, F. \& CICCONE, A. (2004). "Trade and Productivity". The Quarterly Journal of Economics, 119(2), 612-645.

ALONSO, J. (2012). Calidad institucional y entorno empresarial. Mediterráneo Económico, 21, 65-78.

ALONSO, J. Y GARCIMARTIN, C. (2011) "Criterios y Factores de Calidad Institucional: Un Estudio Empírico”. Revista Economía Aplicada XIX. (55)

ÁLVAREZ, R., \& GARCÍA, A. (2010). "Productividad, innovación y exportaciones en la industria manufacturera chilena". 155184.

ANDERSON, J. (1979). "A Theoretical Foundation for the Gravity Equation". American Economic Review, 69(1), 106-116. BARRO, R.J. (1999). "Determinants of Democracy". Journal of Political Economy 107(6), 158-83.

BAYOUMI, T. \& EICHENGREEN, B. (1997). "Exchange Rate 
Volatility and Intervention: Implications of The theory of Optimum Currency Areas". International Monetary Fund and CEPP.

BERGSTRAND, J. (1985). "The Gravity equation in international Trade: Some Microeconomic Foundations and Empirical Evidence". The Review of Economics and Statistics 71, 143153.

BHAGWATI, J. (1978). "Anatomy and Consequences of Exchange Control Regimes". Cambridge: M.A. Ballinger Publishing Co. BORNER, S.; BRUNETTI, A. \& WEDER, B. (1992). "Institutional Obstacles to Latin American Growth". ICEG Occasional Paper 24.

BREUSS, F. \& EGGER, P. (1999). "How Reliable are Estimations of East-West Trade Potentials Based on Cross-Section Gravity Analyses?”. Empírica 26, 81-95.

BUENDÍA, E. (2013). "El Papel de la Ventaja Competitiva en el Desarrollo Económico de los Países. Análisis Económico 69, Vol. XXVIII, 55-78.

CARBALLO, A. (2010). Poverty And Corruption In Latin America: Challenges For A Sustainable Development Strategy". OPERA - Observatorio de Políticas, Ejecución y Resultados de la Administración Pública.(10), 41-65.

CHEN, I. \& WALL, H. (1999). "Controlling for Heterogeneity in Gravity Models of Trade”. Federal Reserve Bank of St. Louis. Working Paper.

CONTRERAS-CASTILLO, J. M. (1999). "La competitividad de las exportaciones mexicanas de aguacate: un análisis cuantitativo". Revista Chapingo Serie Horticultura, 5, 393400.

COULIBALY, S. Y FONTAGÉ, L. (2006). "South-South trade: Geography Matters". Journal of African Economies 15(2), 313-341.

DE GREGORIO REBECO, J. (2005). "Crecimiento económico en Chile: evidencia, fuentes y perspectivas”. Estudios Públicos, (98), 19-86. 
DEGREGORIO,J.(2008). “ELCRECIMIENTOECONÓMICO DE LA AMÉRICA LATINA: Del desencanto del siglo XX a los desafíos del XXI". (Spanish). Trimestre Económico, 75(297), 5-45.

EDISON, H. (2003). “ ¿Vínculos sólidos?: ¡cómo es la relación entre la calidad institucional y el desempeño económico?”. Finanzas y desarrollo: publicación trimestral del Fondo Monetario Internacional y del Banco Mundial, 40(2), 35.

EGGER, P. (2000). "A note on the Proper econometric Specification of Gravity Equation”. Economics Letters 66, 25 -31.

FAJNZYLBER, F. (1988). "Competitividad internacional: evolución y lecciones”. Revista de la CEPAL. 36.

FONSECA, J. A., MUÑÓZ, N. A., \& CLEVES, J. A. (2015). “El sistema de gestión de calidad: elemento para la competitividad y la sostenibilidad de la producción agropecuaria colombiana”. Revista de Investigación Agraria y Ambiental (RIAA, 2(1), 9-22.

FRANKEL, E., \& ROMER, D. (1999). "Does Trade Cause Growth. American Economic Review 89(3), 379-399.

GANTMAN, E. \& DABÓS, M. (2013) "Finanzas, Calidad Institucional y Crecimiento (1961-2005)” Revista Economía Aplicada XXI, (61).

GARCIA RODRÍGUEZ, M., GÓMEZ FRAIZ, J., \& MARTÍNEZ SERNA, A. (2010). Importancia de la marca paraguas y el made in en la competitividad internacional del granito". Cuadernos de Gestion, 10(2), 29-41.

GÓMEZ, Á. M. P. (2013). "El comercio internacional un instrumento para alcanzar el desarrollo de Costa Rica”. Economía y Sociedad, 16(39-40), 109-132.

HAUSMANN, R., HWANG, J., \& RODRIK D. (2006). "What you export matters". CEPR Discussion Paper, No 5444.

HELPMAN, E. \& KRUGMAN, P (1985). "Market Structure and Foreign Trade. Increasing Returns, Imperfect Competition, and the International Economy”. Cambridge, MA. MIT Press (1985-1996). 
HERZER, D., \& NOWAK-LEHMANN, D. (2006a). "Is there a long-run relationship between export and imports in Chile?". Applied Economics Letters 13(15), 981-986.

HERZER, D., \& NOWAK-LEHMANN, D. (2006b). "What does export diversification do for growth? An Econometric Analysis". Applied Economics, 38(15), 1825-1838.

HINOJOSA PÉREZ, A. (2007). "Los desafíos económicos, tecnológicos e institucionales de la sociedad peruana frente a la globalización en el siglo XXI". Revista Alternativa Financiera, 4(4), 68-76.

JIMÉNEZ, F. (2012). "Ciclos y determinantes del crecimiento económico: Perú 1950-1996”. Economía, 20(39-40), 103164.

KOGAN, J. H. (2012). "Infraestructura, integración física y competitividad internacional de la Región Sudamericana". Departamento de Economía, Universidad del Pacífico Chapters of Books, 1, 73-88.

KRAUSE, M. (2007). "Calidad de las instituciones y competencia institucional” .Londres: Internacional Policy Network.

KRUEGER, A. (1978). "Liberalization attempts and consequences". Cambridge MA: Ballinger Publishing Co.

LEON, C.; DE LEON, J. \& ARANA, J. (2014) "Relación entre Corrupción y Satisfacción” Revista Economía Aplicada XXII (64). Primavera.

LEWIS, W. A. (1978). "The Evolution of International Economic Order". Princeton University Press.

LINNEMANN, H. (1966). "An Econometric Study of International Trade Flows". Amsterdam: North Holland publishing Co.

MARTÍNEZ-ZARZOSO, I., \& Doyle, E. (2007). "Trade, Productivity and Institutional Quality: Issue and Empirics". German Development Economics Conference, Göttingen 2007 from Verein für Socialpolitik. Research Committee Development Economics.

MARTO SARGENTO, A. (2007). "Empirical Examination of the Gravity Model in two Different Contexts: Estimation and 
Explanation”. Jahrbuch für Regionalwissenschaft.

MÁTYÁS, L. (1997). "Proper Econometric Specifications of the Gravity Model”. The World Economy 20, 363-69.

MAURO, P. (1995). "Corruption and Growth". Quarterly journal of Economics110, 681-712.

MAYORGA SANCHEZ, J., \& MARTÍNEZ ALDANA, C. (2010). "Paul Krugman y el nuevo comercio internacional". Criterio Libre.

MCMILLAN, M. S., \& RODRIK, D. (2011). "Globalization, structural change and productivity growth (No. w17143)". National Bureau of Economic Research. Working paper $\mathrm{N}^{\circ}$ 17143.

MONCAYO, E. (2004). "El debate sobre la convergencia económica internacional e interregional: enfoques teóricos y evidencia empírica”. EURE (Santiago), 30(90), 7-26.

NAUDE, W., \& GRIES, T. (2009). "Explaining Regional Export Performance in a Developing Country: The Role of Geography and Relative Factor Endowments". Regional Studies, 43(7), 967-979.

NOVELLA, J. (1995). "Mundialización, competitividad, comercio internacional, política industrial y empleo". Afers Internacionals, Vol. 29-30. 89-95. Fundación CIDOB.

NURKSE, R. (1964). "Equilibrio y Crecimiento en la Economia Mundial". ed. Rialp, Madrid.

OLIVARES VALDÉS, O. (2009). "Competitividad y calidad factores críticos que contribuyen al éxito de Empresas de Menor Tamaño en mercados globales de país emergente". Revista Chilena de Economía y Sociedad, 3(1), 31-53.

OVERBAUGH, S. (2013). "National culture, country-level competitiveness, and economic development". International Journal of Business \& Economics Perspectives, 8(1), 93-108.

PARK, J.-Y., \& JANG, S. (2014). "An Extended Gravity Model: Applying Destination Competitiveness". Journal of Travel \& Tourism Marketing, 31(7), 799-816.

PÉREZ DUNIA, R. (2007). "Competitividad Empresarial de cara 
a la exportación. Una conceptualización necesaria”. Economia y Desarrollo, 142(2), 67-79.

PÖYHÖNEN (1963). "A Tentative Model for the Volumen of Trade between Countries". Weltwirtschaftliches Archiv 90, 93-99.

PRICE, J. (2011). “América Latina 2011: Gorda, Contenta Y Poco Competitiva”. Latin Trade, 19(2), 45-46.

RAMÍREZ LOPEZ, L., \& SÁNCHEZ JUÀREZ, I. (2013).

"Crecimiento económico, corrupción e instituciones en México". Nóesis: Revista de Ciencias Sociales y Humanidades, 22(43), 104-133.

REDDING, S., \& VENABLES, A. (2003). "Georgraphy and Export Performance: External market Acces and Internal Suppy Capacity”. NBER Working paper No W9637. Social Science Reseach Network.

RODRÍGUEZ BENAVIDES, D., \& LÓPEZ HERRERA, F. (2010). "Exportaciones y Productividad Laboral Del Sector Manufacturero En México”. Problemas del Desarrollo. Revista Latinoamericana de Economia, 41(161), 41-58.

RODRÍGUEZ BENAVIDES, D., \& VENEGAS-MARTÍNEZ, F. (2011). "Efectos de las exportaciones en el crecimiento economico de Mexico: Un analisis de cointegración, 19292009”. EconoQuantum, 7(2), 55-71.

RODRÍGUEZ-POSE, A., TSELIOS, V., WINKLER, D., \& FAROLE, T. (2013). "Geography and the Determinants of Firm Exports in Indonesia”. World Development, 44, 225240.

RODRIK, D. (2000). Institutions for high-quality growth: what they are and how to acquire them. Studies in Comparative International Development, 35(3), 3-31.

RODRIK, D. (2003). "Growth Strategies”, ed. Harvard University. RODRIK, D. (2005). "Políticas de diversificación económica”. Revista de la CEPAL 87.

RODRIK, D. (2008). "Second-Best Institutions". Amercian Economic Review, 98(2), 100-104. 
RODRIK, D. (2014). “The Past, Present, and Future of Economic Growth". Challenge (05775132), 57(3), 5-39.

RODRIK, D, \& SUBRAMANIAM, A. (2003). "La primacía de las instituciones (y lo que implica)". Finanzas y desarrollo: publicación trimestral del Fondo Monetario Internacional y del Banco Mundial, 40(2), 31.

ROJAS, P., LÓPEZ, E., \& JIMÉNEZ, S. (1997). "Determinantes del crecimiento y estimación del producto potencial en Chile: el rol del comercio". Análisis del Crecimiento en Chile.

ROSALES, O. (2009). "La globalización y los nuevos escenarios del comercio internacional”. REVISTA CEPAL 97.

SACHS, J. \& WARNER, A. (1997). "Sources of slow growth in African economies". Journal of African Economies 6(3), 335376.

SACHS, J. (2000). “Tropical Underdevelopment". CID Working Paper No 57. Center for Interational Development at Harvard University.

SACHS, J. (2003). "Las Instituciones son Importantes, Pero no Para Todo". Finanzas y Desarrollo, Junio, 38-41.

SACHS, J., \& WARNER, A. (1995). "Economic Reform and the Process of Global Integration”. Brookings Papers on Economic Activity, 1-118.

SALA-I-MARTIN, X; OSORIO, BB; BLANKE, J.; DRZENIEK, M.; GEIGER, T. (2011). “The Global Competitiveness Report 2011-2012: Setting the Foundations for Strong Productivity". (en línea). Ed K Schwab. Geneva, CH. SRO-Kundig. 527 p. Disponible en http://www3.weforum.org/docs/WEF_GCR_ Report_2011-12.pdf.

TINBERGEN, J. (1962). "Shaping the World Economy". Suggestions for an International Economic Policy. New York: Twentieth Cetury Fund.

VALENZUELA-KLAGGES, B. (2011). "Comercio y calidad institucional en el crecimiento económico del cono sur americano: cuestiones y desafíos". Revista Pilquen-Sección Ciencias Sociales, 13(14), 1-8. 
YEVENES SUBIABRE, A., \& ANDALAFT CHACUR, A. (2006). "Competitividad Territorial Y Estrategia Empresarial: El Caso De La Industria Forestal Exportadora De La Región Del Bío Bío”. Horizontes Empresariales, 5(1), 39-51.

Fecha de Recepción del Artículo: 25 de julio de 2016 FECHA DE ACEptación: 21 de noviembre de 2016 